\title{
ESTIMACIÓN DEL CONTENIDO DE BIOMASA, FIJACIÓN DE CARBONO Y SERVICIOS AMBIENTALES, EN UN ÁREA DE BOSQUE PRIMARIO EN EL RESGUARDO INDÍGENA PIAPOCO CHIGÜIRO-CHÁTARE DE BARRANCOMINAS, DEPARTAMENTO DEL GUAINÍA (COLOMBIA)
}

\author{
Nubia Janeth Quiceno Urbina ${ }^{1}$ \\ Gloria Mónica Tangarife Marín ${ }^{2}$ \\ Ricardo Álvarez León ${ }^{3}$
}

Recibido el 7 de enero de 2015, aprobado el 27 de octubre de 2015 y actualizado el 19 de mayo de 2016

DOI: 10.17151/luaz.2016.43.9

\section{RESUMEN}

En Barrancominas (Guainía, Colombia) las diferentes formas culturales de trabajo y producción de la comunidad Piapoco, incluyen la tumba y quema de bosques a orillas del río, para ampliación de frontera agrícola, establecimiento de cultivos ilícitos y potreros; lo cual contribuye en el aumento de las concentraciones de carbono en la atmósfera que, a la vez, repercute en el cambio climático global. En consecuencia, el presente estudio quiso estimar el contenido de biomasa, fijación de carbono y los servicios ambientales, en un área de bosque primario en el Resguardo Indígena Chigüiro-Chátare, empleando una metodología no destructiva. Se estimó tanto la biomasa aérea, como el contenido de carbono (C) del estrato arbóreo del bosque por hectárea y la tasa de producción de hojarasca de las especies maderables: Mure, Pendare y Arenillo blanco. La biomasa aérea fue estimada a partir de los parámetros medidos en campo como altura y DAP y densidades de la madera de cada especie. Además, se analizó la dominancia de las especies para de esta forma conocer la representatividad del aporte de biomasa aérea y contenido de carbono en el bosque.

\section{PALABRAS CLAVE}

Couma sp., Qualea paraensis, Cedrelinga cateniformis, biomasa aérea, biomasa foliar, fijación de carbono, bosque primario, Guainía, Colombia. 


\title{
ESTIMATED BIOMASS CONTENT, CARBON SEQUESTRATION AND ENVIRONMENTAL SERVICES, IN A FOREST AREA IN THE SHELTER PRIMARY INDIAN PIAPOCO CHIGÜIRO-CHÁTARE, BARRANCOMINAS, GUAINIA DEPARTMENT (COLOMBIA)
}

\begin{abstract}
In Barrancominas (Guainía) different cultural forms of work and production of the Piapoco community, includes the slash and burn of forest along the river, to extend the farming, establishment of illicit crops and pastures contribute to increase carbon concentrations in the atmosphere which in turn affect global climate change. Thus this study wanted to value the biomass content, carbon fixation and ecosystem services in an area of primary forest in the indigenous community of ChigüiroChátare using a nondestructive method or indirect. In this study the aerial biomass was estimated as well as the carbon (C) content of the arboreal stratum of forest per hectare and production rate of dead leaves of three species forestry Mure, Pendare and Arenillo trees. The aerial biomass was estimated from the measured parameters in field such as: height, DAP and wood densities of each species. In addition, the species dominance was analyzed in order to know the representativeness of the biomass contribution and carbon content in the forest.
\end{abstract}

\section{KEY WORDS}

Couma sp., Qualea paraensis, Cedrelinga cateniformis, biomass aerial, biomass foliar, carbon fixation, primary forest, Guainía, Colombia.

\section{INTRODUCCIÓN}

El aumento de la concentración de dióxido de carbono (CO2) en la atmósfera de la tierra es una preocupación mundial y se considera como uno de los seis principales gases que intervienen en el efecto invernadero (GEI), el cual está contribuyendo en el cambio climático (Concha et al., 2007).

En Barrancominas (Guainía) las formas culturales de trabajo y producción de la comunidad Piapoco incluyen la tumba y quema de bosques a orillas del río, para ampliación de frontera agrícola, establecimiento de cultivos ilícitos y potreros; lo cual deteriora la calidad del aire, que afecta la capacidad de este territorio para 
continuar siendo una válvula global de oxígeno, carbón y agua necesaria para seguir viviendo.

Sumado a lo anterior, en el resguardo de la comunidad indígena se ve modificado el paisaje, debido a la disminución de las especies maderables y no maderables, afectación del río Guaviare, extinción de especies forestales nativas que perjudican a la vez la fauna asociada a la vegetación; lo anterior se deriva de ausencia de políticas, estrategias y programas de capacitación en educación ambiental y aprovechamiento de recursos naturales en la zona.

También, se observan fenómenos de erosión como la pérdida de la capa superficial del suelo, deslizamiento de tierra a orillas del río Guaviare, reducción del caudal de caños y esterilidad del suelo; producto de la mala situación económica que obliga a la tala para construcción de casas, senderos, bongos, obtención de leña y suplir la demanda de maderas comerciales.

Esta contribución de estimación, almacenamiento de carbono e identificación de servicios ambientales era una necesidad local, que requerirá de atención especial y urgente para lograr un papel preponderante y activo que genere capacidades en la comunidad para comprender los múltiples beneficios que se pueden generar como regulación del clima, prevención de desastres, disminución de la presión al bosque natural, protección de cuencas hidrográficas, conservación del paisaje y biodiversidad, además de convertirse en una oportunidad social y económica a través de la venta de servicios ambientales.

\section{MATERIALES Y MÉTODOS}

\section{Área de estudio}

El Resguardo Indígena Piapoco Chigüiro-Chátare tiene 18.320 ha, dista $4 \mathrm{~km}$ terrestres o $36 \mathrm{~km}$ fluviales de la comunidad de Barrancominas situada al noroccidente del departamento del Guainía, aproximadamente a $220 \mathrm{~km}$ de distancia de su capital (Puerto Inírida), con una localización entre los 02 10'17" y $03^{\circ} 29^{\prime} 00^{\prime \prime} \mathrm{N}$ y entre los $66^{\circ} 50^{\prime} 44^{\prime \prime}$ y $69^{\circ} 48^{\prime} 38^{\prime \prime}$ W. 
Su ubicación geográfica regional corresponde a la Amazonia septentrional colombiana. El área pertenece a la zona de vida bosque muy húmedo tropical (bmh-T) según la clasificación de Holdridge (Zambrano et al., 2006, p. 68).

\section{Materiales}

Los materiales usados en las actividades de campo fueron: en el Inventario Forestal: un GPS, dos cintras métricas de $50 \mathrm{~m}$, Instrumento Blume Leiss, 3 machetes, estacas de madera (pueden conseguirse en el campo), cinta amarilla peligro, pintura amarilla brillante, marcadores, lápiz, formularios, cámara digital; y en el Cálculo de Biomasa: una cinta métrica, cuatro machetes, una balanza de 50 kg, 1 balanza de 1 a $50 \mathrm{~kg}$ con una precisión de un gramo, bolsas plásticas y de papel, marcadores, lápiz, formularios y cuadro con angeo plástico, lona y alambre para elaborar trampas, solución fijadora y protectora.

En la presente investigación se estudió un área de bosque primario en el Resguardo Indígena Piapoco Chigüiro-Chátare. Se estimó tanto la biomasa aérea, como el contenido de carbono $(\mathrm{C})$ del estrato arbóreo del bosque por hectárea y la tasa de producción de hojarasca del Mure (Cedrelinga cateniformis), Pendare (Couma sp.) y Arenillo blanco (Qualea paraensis).

Además, se analizó la dominancia de las especies para conocer la representatividad del aporte de biomasa aérea y el contenido de carbono en el bosque. El estudio se dividió en tres fases: fase de campo, fase de laboratorio y fase de análisis de datos.

\section{Fase de campo}

Debido al enfoque conservacionista de esta investigación y a limitaciones de tiempo y recursos, se utilizó el método no destructivo para el estudio de la biomasa y contenido de carbono de un área de bosque primario como se detalla a continuación:

Cartografía social: Empleando la herramienta de cartografía social de Rodríguez et al. (1999) se elaboraron los mapas del departamento para ubicar el corregimiento de Barrancominas, posteriormente en otro mapa a mayor detalle ubicar el Resguardo Chigüiro-Chátare y finalmente la zona de estudio, también se determinaron las especies de importancia para la comunidad y el área de 
muestreo, siempre con el apoyo de los líderes de la comunidad: el Capitán, el Líder, el Pastor, el ExCapitán y el Director de la Escuela Agapito Sandoval.

Selección de área de muestreo: La metodología desarrollada se basó en la guía de Rügnitz et al. (2009) para la determinación de carbono en pequeñas propiedades rurales del ICRAF (International Centre for Research in Agroforestry), la cual establece para la planificación de mediciones en campo las siguientes etapas: 1) Definición del área de cobertura del proyecto. 2) Estratificación de las áreas del proyecto. 3) Decisión sobre cuál depósito de carbono a medir. 4) Determinación del tipo y número de parcelas. 5) Determinación de la frecuencia de las mediciones.

Cabe resaltar que para desarrollar el proyecto fue necesario contar con la aprobación de la máxima autoridad indígena de la comunidad Chigüiro-Chátare: el Capitán; el cual, al ver la importancia de la misma, autorizó y sugirió verbalmente que se contara con el apoyo de los líderes comunitarios, los cuales estuvieron presentes durante las salidas de campo colaborando con las actividades requeridas durante la investigación.

\section{Establecimiento de parcelas}

Localización: Se establecieron parcelas permanentes, las cuales fueron localizadas sobre suelo homogéneo, con adecuado acceso, protegidas de la perturbación humana y bajo el cuidado de la comunidad.

Posición: Las parcelas se localizaron en el lugar donde se observan "manchas" de alguna de las 3 especies seleccionadas por la comunidad.

Tiempo: Aproximadamente desde la comunidad de Chigüiro hasta el lugar de las parcelas fue de una hora y 15 minutos; y para delimitar una parcela fue de 40 minutos.

Forma: Se establecieron parcelas cuadradas por ser más prácticas de estudiar que las circulares y tener menos periferia y área radial que las parcelas rectangulares, por tanto se tienen menos problemas para decidir sobre la presencia de árboles dentro o fuera de los límites de la parcela.

Tamaño: Se estableció un área efectiva de 1,5 ha, con 6 parcelas de 50 × $50 \mathrm{~m}$. Pinelo (2000) recomienda que las parcelas permanentes de medición (PPM) en el 
bosque tropical tengan una forma cuadrada debido al menor perímetro con respecto a parcelas rectangulares y con un área de 0,25 ha.

Visibilidad: Para ubicación de las parcelas en las cuatro esquinas se colocaron estacas obtenidas en el área de estudio, bien enterradas en el suelo, con aproximadamente $80 \mathrm{~cm}$ visibles sobre la superficie del suelo.

Orientación: Se georreferenciaron con GPS, y con ayuda de una brújula se determinó el centro de cada parcela y cada uno de los individuos inventariados.

Demarcado de la parcela con cuerda: Para demarcar fue necesario contar con la ayuda de 3 personas: una con la brújula, una para medir la distancia y otra para extender la cuerda.

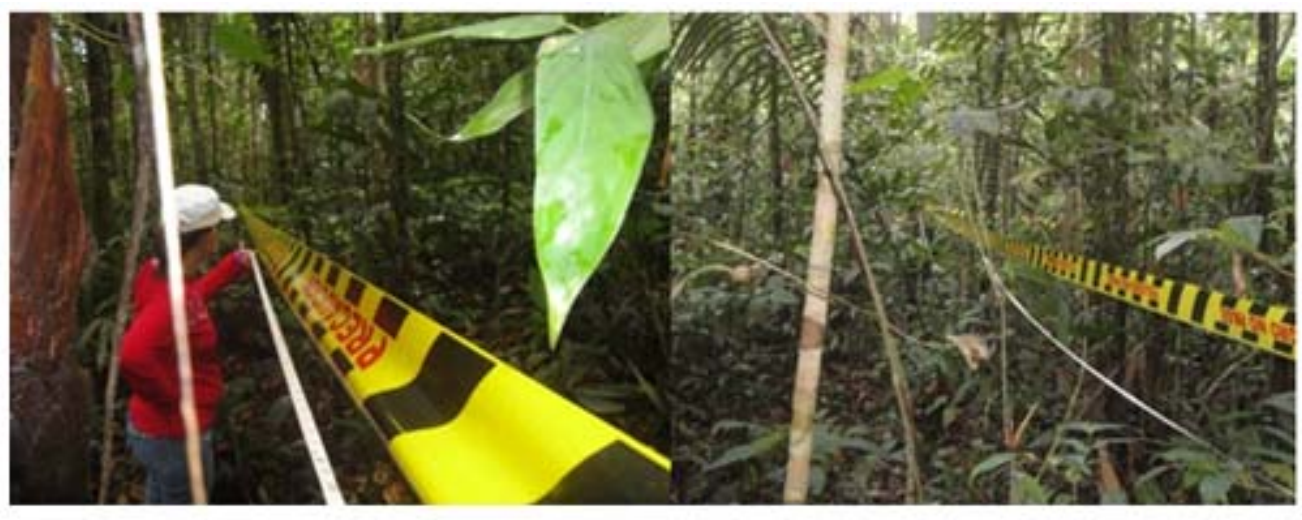

Figura 1. Demarcando parcelas de $50 \times 50 \mathrm{~m}$.

Para el marcado y numeración de los árboles, según Manzanero (2003), se procedió así:

\section{Registros y variables de medición}

a) Número consecutivo: En el formulario de campo, a cada árbol se le asignó un número consecutivo que aparece registrado en el formulario. Este número, aunque el árbol muera o se corte, siempre lo identificará y a ningún otro árbol que ingrese posteriormente podrá asignársele el mismo número.

b) Marcado: Para efectos de una mejor identificación en el campo, cada árbol fue marcado con pintura amarilla brillante, utilizando para ello dos números: arriba el 
número de la parcela y abajo el número consecutivo del árbol, los cuales fueron anotados en el formulario.

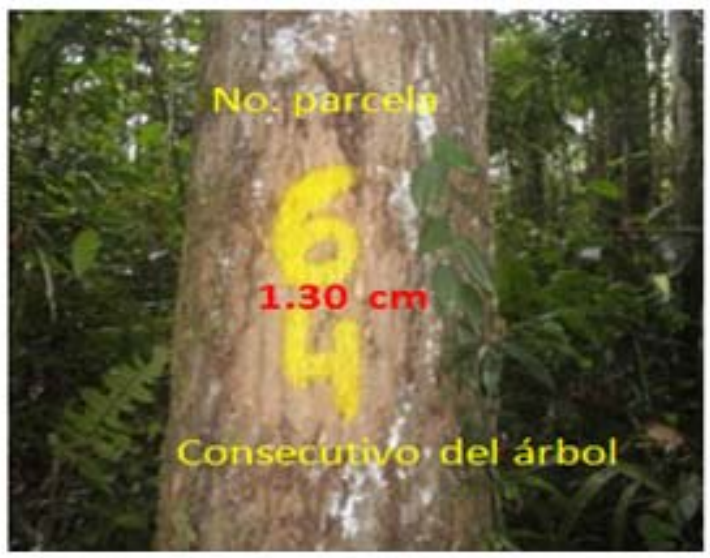

Figura 2. Marcado y numeración de los árboles.

Registros y variables de medición: Para tomar los datos se elaboró una boleta de campo que contiene datos como: No. de árbol, No. de parcela, nombre común, DAP, altura total, altura de fuste, calidad de fuste, forma de la copa, presencia de lianas y observaciones. Además de datos generales como fecha, responsables y datos del sitio.

Diámetro a la altura del pecho (DAP): Esta medida se tomó a los 1,30 m de altura desde el suelo para cada uno de los individuos, con una cinta métrica flexible en donde se obtuvo el perímetro o circunferencia (CAP) que fue convertido en DAP a partir de la fórmula de Villareal et al. (2004):

$$
\mathrm{DAP}=\mathrm{CAP} / \pi
$$

Se midió en una parte libre de daños y defectos, con ayuda de una baliza de 1,30 $\mathrm{m}$ de longitud. Ante la presencia de musgos y lianas en algunos árboles fue necesario retirarlos con el objetivo de no afectar la medida que se registró. 


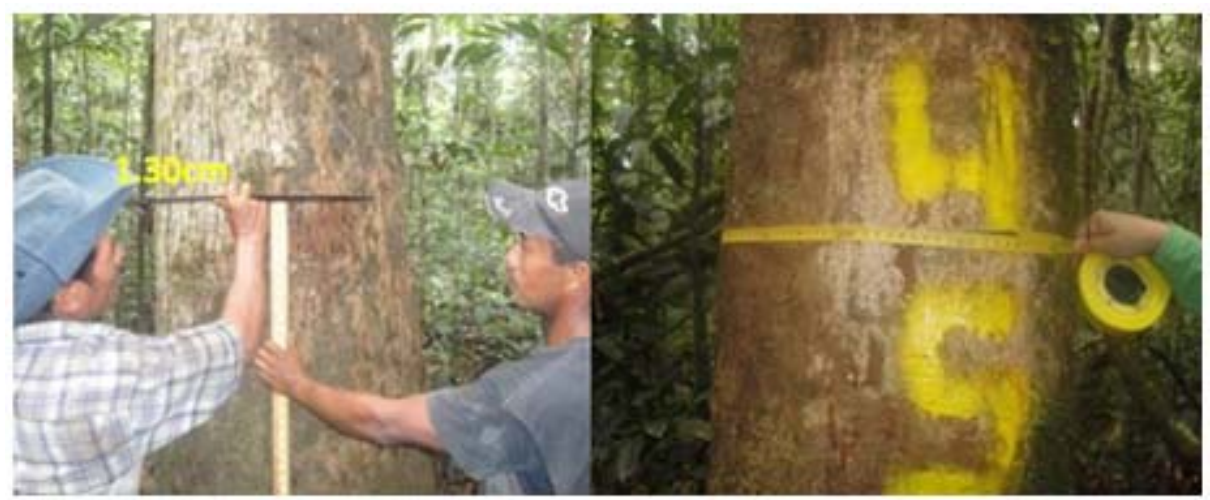

Figura 3. Procedimiento para medir DAP.

Altura total: Debido a las limitaciones del estudio y a la carencia de hipsómetros, se realizó una estimación usando el fundamento trigonométrico Blume-Leiss (Domínguez, 2010), y se acudió también a la ayuda de guías locales con experiencia en estas mediciones en cada una de las parcelas. Esta altura se estimó desde el suelo hasta la cima de la copa de cada individuo (Vallejoet al., 2005).
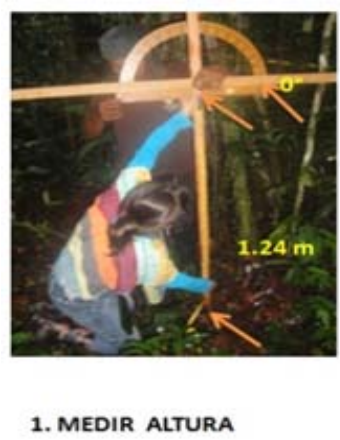

Desde el suelo hasta el punto de origen de la mira

Se observa por el borde interno de la mira hasta visualizar la copa del árbol

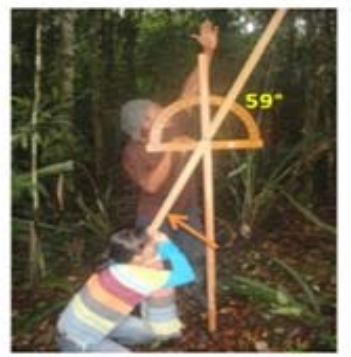

2. CALCULAR EL ANGULO

\section{MEDIR DISTANCIA}

Desde el árbol hasta el punto de origen de la mira

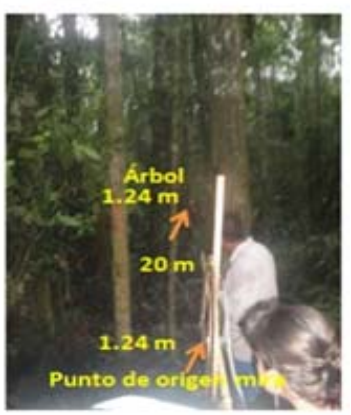

Figura 4. Procedimiento para calcular altura total.

\section{Registros indispensables en las parcelas}

Nombre común: Se recurrió al conocimiento empírico de la comunidad, la cual identificó las diferentes hojas de la Figura 5. Posteriormente se usó un listado 
confiable de especies arbóreas de la comunidad Chigüiro-Chátare según Pedraza et al. (s.f.) para asignar los nombres científicos.

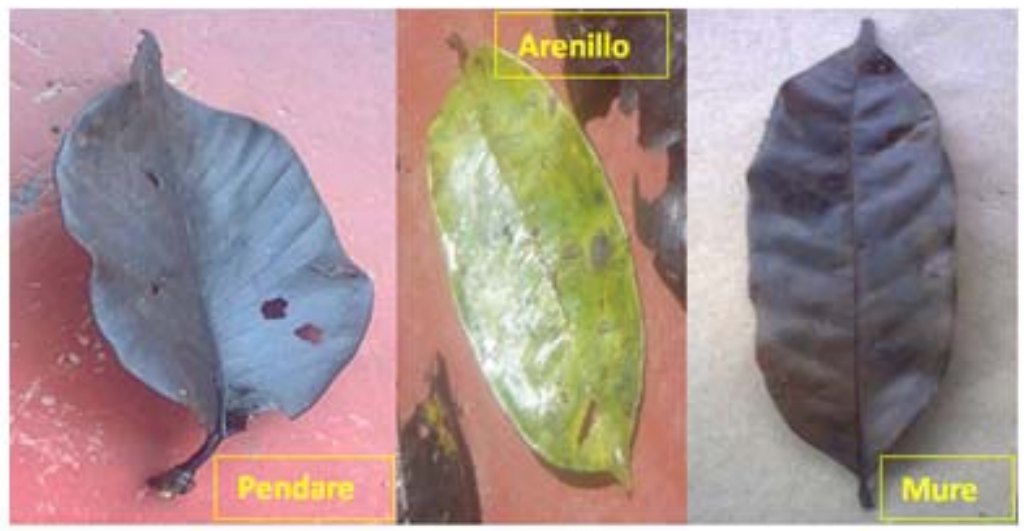

Figura 5. Hojas de las tres especies con base en el conocimiento empírico.

Diámetro del fuste: Los diámetros se midieron al milímetro completo. Se usaron cintas diamétricas estables y resistentes al trato duro y a la humedad tropical. Se redondeó al milímetro inferior, ya que se considera como un error sistemático que puede ser ignorado (Synnott, 1990). 


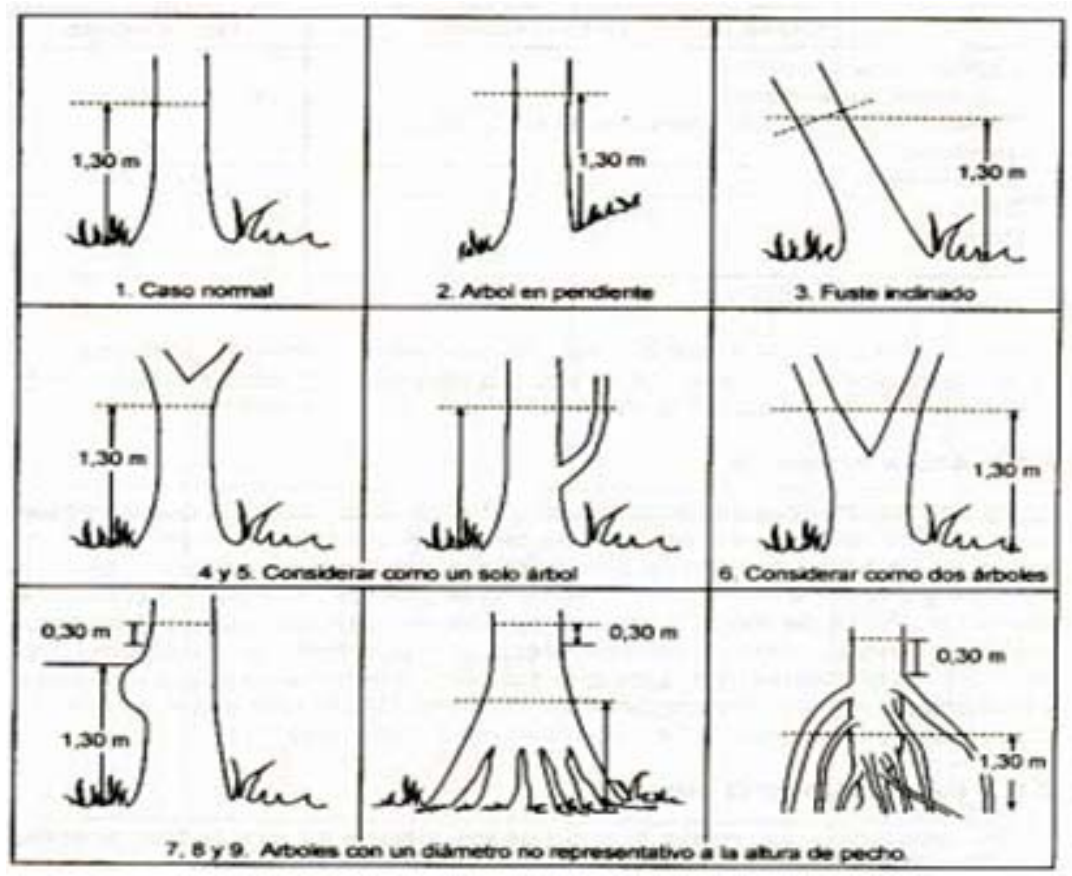

Figura 6. Recomendaciones para medir el diámetro de un árbol, según CATIE (1994).

Calidad de fuste: Se calificó según los códigos que aparecen en la Tabla 1 y bajo el criterio de los lugareños, quienes conocen la calidad comercial o no de las especies.

Tabla 1. Códigos para determinar clase de calidad de fuste, según Hutchinson (1992)

\begin{tabular}{|l|c|}
\hline \multicolumn{1}{|c|}{ Clase de calidad de fuste } & Código \\
\hline Comercial actualmente & 1 \\
\hline Comercial en el futuro & 2 \\
\hline Comercial en el futuro pero con la base podrida (quemado) & 3 \\
\hline Deformado & 4 \\
\hline Dañado & 5 \\
\hline Podrido & 6 \\
\hline
\end{tabular}

Forma de copa: Se empleó el sistema de calificación de cinco puntos como se muestra en la tabla Synnott (1990) y se comparó la observación en campo con las formas de la copa que aparecen en la figura. 
Forma de copa: Se empleó el sistema de calificación de cinco puntos como se muestra en laTabla 2 Synnott (1990) y se comparó la observación en campo con las formas de la copa que aparecen en la Figura 7.
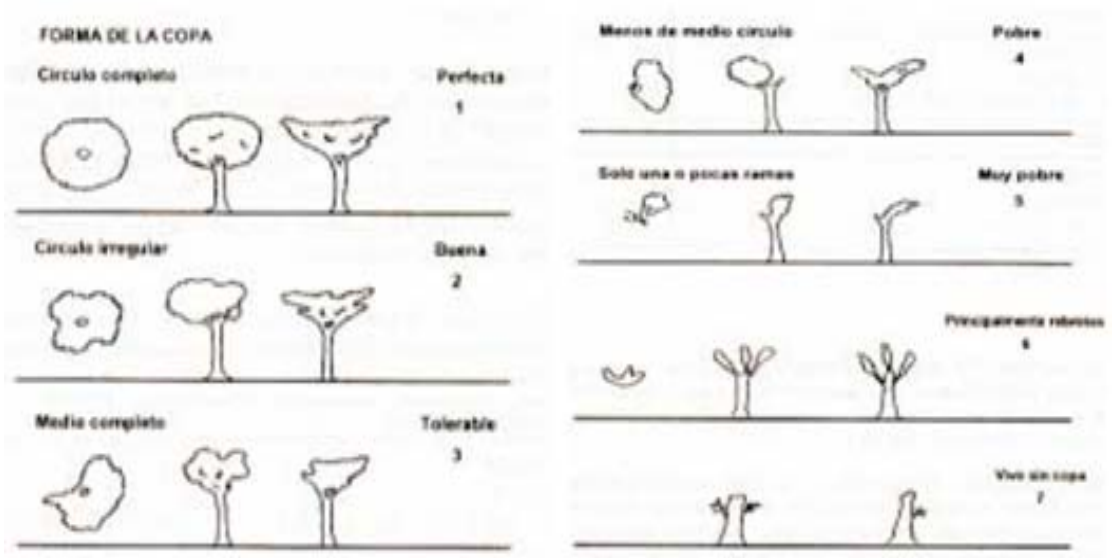

Figura 7. Calificación de la forma de la copa, según Synnott (1990).

Tabla 2. Clasificación de forma de la copa, según Synnott (1990).

\begin{tabular}{|l|c|}
\hline \multicolumn{1}{|c|}{ Forma de la copa } & Código \\
\hline Copa vigorosa, círculo completo o irregular (1 y 2) & 1 \\
\hline Medio círculo (3) & 2 \\
\hline Menos de medio círculo (categorías 4 y 5) & 3 \\
\hline Solo rebrotes o sin copa (categorías 6 y 7) & 4 \\
\hline
\end{tabular}

Infestación por trepadoras: Se observó en campo y se calificó teniendo en cuenta los códigos que aparecen en la Tabla 3. 
Tabla 3. Códigos para calificar presencia de lianas, según Synnott (1990)

\begin{tabular}{|l|c|}
\hline \multicolumn{1}{|c|}{ Presencia de lianas } & Código \\
\hline No visible en fuste y copa & 1 \\
\hline Presente en fuste, no existe conexión con otros árboles & 2 \\
\hline Presente en fuste, existe conexión con otros árboles & 3 \\
\hline Presente en copa, no existe conexión con otros árboles & 4 \\
\hline Presente en copa, existe conexión con otros árboles & 5 \\
\hline Presente en fuste y copa, no existe conexión & 6 \\
\hline Presente en fuste y copa, existe conexión & 7 \\
\hline
\end{tabular}

Colecta de hojarasca: Para la estimación de la hojarasca se usó el método de cosecha, para lo cual se utilizaron trampas de hojarasca que consisten en una sección circular (para disminuir el efecto de borde) de $0,25 \mathrm{~m}^{2}$ de área, donde cada colector tiene un área de colección de 0,78 $\mathrm{m}^{2}$ aproximadamente (Salas e Infante, 2006; Vargas y Varela, 2007).

Inicialmente se situaron 36 trampas, distribuidas en 6 trampas por parcela, 3 por cada árbol y 2 árboles en cada parcela. Los árboles fueron seleccionados teniendo en cuenta que al final se muestrearan 4 árboles de cada una de las 3 especies objeto de estudio, se incluyó árboles adultos y juveniles; este tipo de muestreos es aconsejable en bosques de edades mixtas con el fin de controlar la variable de edad en la productividad de las especies y, de esta forma, tener en cuenta la heterogeneidad del bosque (Bonham, 1989).

Las trampas se dejaron instaladas por un periodo de 15 días, al cabo de los cuales se recolectó el material de las estructuras no renovables (Salas e Infante, 2006). En vista de la escasa hojarasca colectada con las primeras trampas se optó por ampliar dicho diámetro usando lona, esta vez se colocaron dos trampas por árbol (mismos árboles de la primera colecta) con una dimensión de $2 \mathrm{~m} \times 2 \mathrm{~m}$. 

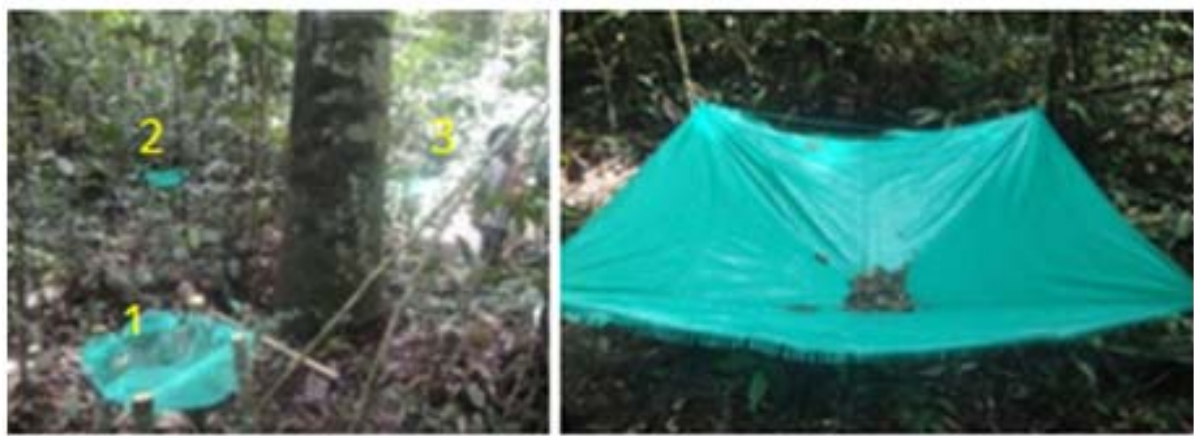

Figura 8. Ubicación y modelo inicial y final de trampa de hojarasca.

Las muestras recolectadas se seleccionaron, pesaron en fresco y se guardaron en bolsas de papel con aireación suficiente para presecarlas y evitar la pérdida por descomposición, luego fueron nuevamente pesadas y se almacenaron para llevarlas a los procedimientos de laboratorio.
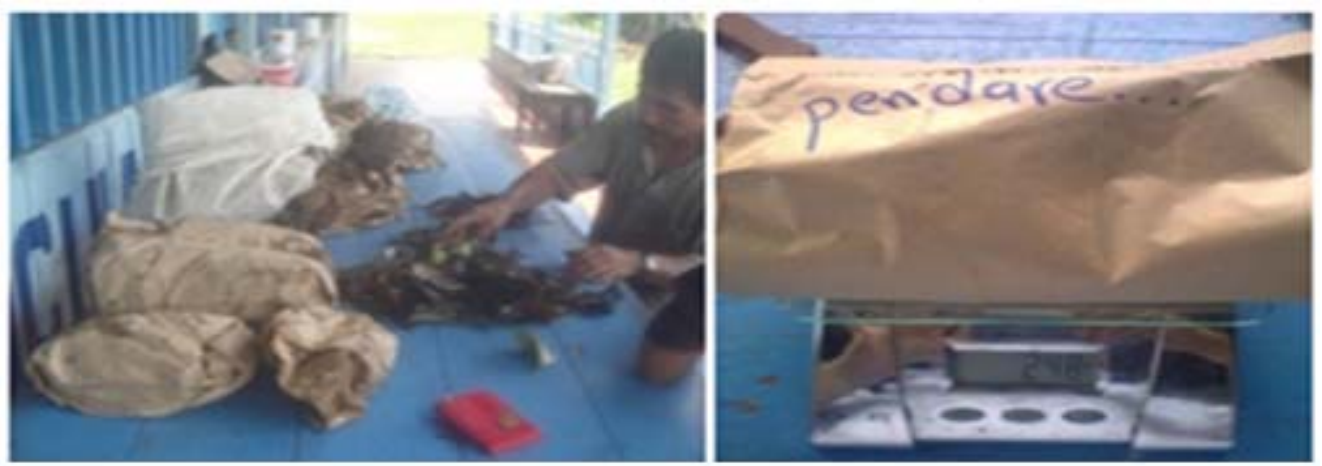

Figura 9. Selección y pesaje en fresco de muestras.

Observación de fauna y flora: Se realizó observación directa e identificación in situ de individuos durante las salidas, adicionalmente se obtuvo información de los usos más frecuentes por parte de los miembros de la comunidad durante las salidas de campo.

\section{Fase de laboratorio}

Con las muestras colectadas en campo se continuó el proceso en el Laboratorio de Nutrición Animal de la Universidad de los Llanos en Villavicencio (Meta). 
- A las submuestras de las hojas de las tres especies se les realizó análisis bromatológico.

- Cada una de las submuestras de hojarasca se secó en un horno a $70^{\circ} \mathrm{C}$ durante 3 días, y se pesaron las submuestras para obtener el aporte de biomasa en peso seco. El peso se tomó por cada especie arbórea por separado, únicamente se muestrearon hojas.

\section{Fase de análisis de datos}

La información de campo y laboratorio se registró en una tabla de base de datos en Excel, donde se documentó el nombre de las especies y estudio bromatológico realizado en la Universidad de los Llanos, el DAP y alturas respectivos para cada especie estudiada. Con esta base de datos se prosiguió al cálculo de biomasa aérea de la siguiente forma:

Estimación de biomasa aérea: Para estimar la biomasa aérea se utilizaron las 3 especies arbóreas con un DAP mayor a $10 \mathrm{~cm}$, de acuerdo a Brown (2002) los árboles de diámetros menores contribuyen poco a la biomasa y carbono de un bosque. Se utilizó una ecuación propuesta por Brown, la cual será descrita a continuación:

$$
\mathrm{BT}(\mathrm{ha})=\mathrm{V}(\mathrm{ha}){ }^{*} \mathrm{DP}(\mathrm{ha}){ }^{*} \mathrm{FEB}(\mathrm{ha})
$$

BT = Biomasa aérea total.

$V=$ Sumatoria del volumen de todas las especies.

$\mathrm{DP}=$ Densidad promedio.

FEB $=$ Factor de expansión de biomasa.

- Volumen (V): A partir de los datos dasométricos tomados en los árboles de las parcelas se calcula el volumen comercial; para especies latifoliadas la siguiente fórmula:

$$
V=1 / 4(3.1416)^{*} \mathrm{DAP} 2{ }^{*} \mathrm{hf}{ }^{*} \mathrm{Ff}
$$

DAP = Diámetro Altura de Pecho (1,3 m del suelo).

$\mathrm{Hf}=$ Altura fustal $(\mathrm{m})$.

$\mathrm{Ff}=$ Factor de forma $(0,70$ en latifoliada y 0,47 en pino $)$. 
El volumen obtenido para las parcelas, se suma y se extrapola a una hectárea para aplicarlo en la ecuación propuesta por Brown (1997).

- Densidad promedio (DP): La densidad promedio se calcula a partir de la sumatoria de la proporción del volumen por especie multiplicándolo por la densidad de cada especie como se detalla a continuación:

$$
\mathrm{DP}=\left[(\mathrm{V} 1 / \mathrm{Vt})^{*} \mathrm{D} 1+(\mathrm{V} 2 / \mathrm{Vt})^{*} \mathrm{D} 2+(\mathrm{V} 3 / \mathrm{Vt})^{*} \mathrm{D} 3+\ldots(\mathrm{VnVt})^{*} \mathrm{Dn}\right]
$$

- Factor de expansión de biomasa (FEB): Es la relación entre la biomasa aérea total por hectárea y la biomasa aérea fustal, estimada a partir de los datos de volumen, y se calculó con la fórmula siguiente:

$$
\mathrm{FEB}=[3.213-0.506 \mathrm{Ln}(\mathrm{BV}) \text { para BV mayor a } 190 \mathrm{t} / \mathrm{ha}
$$

Una constante de 1,74 si la BV es menos o igual que $190 \mathrm{t} / \mathrm{ha}$, donde:

$\mathrm{BV}=$ es la biomasa del volumen en $\mathrm{t} / \mathrm{ha}$, calculada como el producto de $\mathrm{V}\left(\mathrm{m}^{3} / \mathrm{ha}\right) \mathrm{y}$ la densidad $\left(\mathrm{t} / \mathrm{m}^{3}\right)$.

Estimación indirecta del contenido de carbono en la biomasa aérea: Para la estimación indirecta por hectárea del contenido de carbono en la biomasa aérea del bosque de roble, se tiene estimado que aproximadamente el $50 \%$ de la biomasa vegetal corresponde al carbono, por lo cual para estimar el carbono almacenado total se multiplicó la biomasa total (BT) por el factor 0,5 en ausencia de información específica.

$$
\mathrm{CBT}=\mathrm{BT}^{*} 0.5
$$

Dónde: $\mathrm{CBT}=$ carbono almacenado (ton/ha), BT = biomasa total (ton/ha).

Producción de hojarasca: Con los datos del peso seco de la hojarasca obtenida en cada una de los muestreos, se midió la tasa de producción de hojarasca, calculada como la relación entre la cantidad producida de hojarasca en un área (trampas de 0,25 $\mathrm{m}^{3}$ y $2 \mathrm{~m}^{2}$ ) en un tiempo de 15 días por muestreo. Se calculó el aporte de hojarasca por cada especie, las épocas del año de muestreo y se analizó la información obtenida (Agudelo, 2009). 
De la muestra llevada (expresada en peso presecado) al laboratorio se utilizó para llevar a la mufla $20 \mathrm{~g}$, de tal forma la información obtenida se recibió en \% del peso presecado. Por tanto, los datos de la Tabla 12 se extrapolaron al peso fresco o in situ.

\section{Índice de Valor de Importancia de las Plantas (IVI)}

Es un parámetro que revela la importancia ecológica relativa de cada especie en una comunidad vegetal. La suma total de los valores relativos de cada parámetro debe ser igual a 100. Por lo tanto, la suma total de los valores del IVI debe ser igual a 300 (Villareal et al., 2004).

\section{RESULTADOS Y DISCUSIÓN}

\section{Construcción de mapas}

La zona de estudio es un terreno denominado tierra firme, lugar donde es posible apreciar "manchas" donde se distingue el Mure, Pendare y Arenillo blanco. El terreno es una masa natural ya que no presenta ninguna intervención humana, no satisface ninguna demanda social y tampoco se trata para silvicultura, lo cual permitió establecer las parcelas sin temor a intervención por el tiempo que duró la investigación.
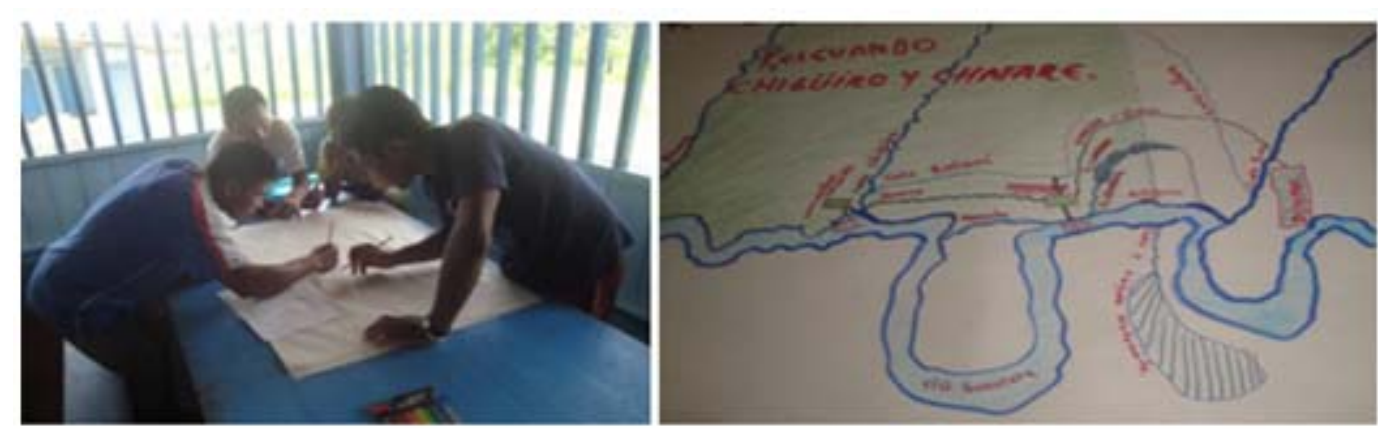

Figura 10. Construcción de mapas del Resguardo Chigüiro-Chátare, comunidad y área de estudio con los líderes de la comunidad. 
El mapa da una idea de quiénes somos, qué tenemos, qué hemos perdido y qué queremos. Es una construcción colectiva donde la mayor ganancia es la recuperación y transmisión de saberes sobre el territorio. Esta metodología acepta que cada persona tiene conocimientos valiosos, sin importar edad, idioma, religión, formación o sexo.

\section{Determinación de las especies}

Tabla 4. Especies determinadas por líderes de la comunidad como de mayor importancia

\begin{tabular}{|c|l|l|l|l|}
\hline $\begin{array}{c}\text { Nombre } \\
\text { común }\end{array}$ & $\begin{array}{c}\text { Nombre } \\
\text { (lengua } \\
\text { piapoco) }\end{array}$ & Familia & $\begin{array}{c}\text { Nombre } \\
\text { cientifico }\end{array}$ & \multicolumn{1}{|c|}{ Características } \\
\hline Muli & Mimosaceae & $\begin{array}{l}\text { Cedrelinga } \\
\text { cateniformis }\end{array}$ & $\begin{array}{l}\text { Es muy trabajable y tiene amplio } \\
\text { mercado en el departamento para } \\
\text { construcción, carpinteria y } \\
\text { ebanisteria. Madera de gran } \\
\text { durabilidad, semidura de color } \\
\text { blanquecino o rosado. }\end{array}$ \\
\hline Pendare & Tzuzi & Apocinaceae & Couma sp. & $\begin{array}{l}\text { Se utiliza principalmente para } \\
\text { extraer una resina (leche) para } \\
\text { impermeabilizar canoas o bongos. } \\
\text { También se sacan tablas livianas } \\
\text { para cielo raso. Su fruto (pepa) es } \\
\text { comestible. }\end{array}$ \\
\hline Klanco & Kálima & Vochysiaceae & $\begin{array}{l}\text { Qualea } \\
\text { paraensis }\end{array}$ & $\begin{array}{l}\text { Seusa para construcciones intemas } \\
\text { y externas. Se sacan estantillos de } \\
5 x \text { 5 10 x 10. Existen varias } \\
\text { especies de arenillo: rosado, } \\
\text { amarillo y blanco. }\end{array}$ \\
\hline Arenillo & & &
\end{tabular}

Tabla 5. Lista de las especies estudiadas

\begin{tabular}{|l|l|l|c|}
\hline Nombre común & \multicolumn{1}{|c|}{ Nombre científico } & Familia & No. de individuos \\
\hline Mure & Cedrelinga cateniformis & Mimosaceae & 4 \\
\hline Pendare & Couma sp. & Apocynaceae & 6 \\
\hline Arenillo blanco & Qualea paraensis & Vochysiaceae & 29 \\
\hline TOTAL & \multicolumn{2}{|l}{} & 39 \\
\hline
\end{tabular}


En el área de muestreo, $2500 \mathrm{~m}^{2}$ distribuidos en 6 parcelas de $50 \times 50 \mathrm{~m}^{2}$, se levantaron todos los árboles pertenecientes a las especies Mure, Pendare y Arenillo blanco con DAP mayor a $20 \mathrm{~cm}$, encontrándose un número total de 39 árboles.

Tabla 6. Frecuencia de las especies

\begin{tabular}{|l|c|c|c|c|c|c|c|}
\hline \multirow{2}{*}{ Especies } & \multicolumn{6}{|c|}{ No. de las Parcelas } \\
\cline { 2 - 7 } & 1 & 2 & 3 & 4 & 5 & 6 & Total \\
\hline Mure & 1 & 0 & 0 & 1 & 0 & 2 & 4 \\
\hline Pendare & 0 & 2 & 0 & 0 & 3 & 1 & 6 \\
\hline Arenillo blanco & 2 & 2 & 11 & 5 & 7 & 2 & 29 \\
\hline Total & 3 & 4 & 11 & 6 & 10 & 5 & 39 \\
\hline
\end{tabular}

La Tabla 6 muestra las especies presentes en cada parcela, se evidencia que el Arenillo blanco está presente en todas las parcelas y que para el caso del Mure y el Pendare solo se encuentran en el $50 \%$ de las parcelas estudiadas.

Tabla 7. İndice de Valor de Importancia

\begin{tabular}{|c|c|c|c|c|c|c|c|}
\hline Especies & $\begin{array}{c}\text { Abundancia } \\
\text { absoluta }\end{array}$ & $\begin{array}{c}\text { Abundancia } \\
\text { relativa }\end{array}$ & $\begin{array}{c}\text { Frecuencia } \\
\text { absoluta }\end{array}$ & $\begin{array}{c}\text { Frecuencia } \\
\text { relativa }\end{array}$ & $\begin{array}{c}\text { Area } \\
\text { basal } \\
(\mathrm{m})\end{array}$ & $\begin{array}{c}\text { Dominancia } \\
\text { relativa }\end{array}$ & IVI \\
\hline Mure & 4 & 10,26 & 50 & 25 & 2,60 & 17,60 & 52,86 \\
\hline Pendare & 6 & 15,38 & 50 & 25 & 3,27 & 21,76 & 62,14 \\
\hline Arenillo blanco & 29 & 74,36 & 100 & 50 & 9,03 & 60,64 & 185,00 \\
\hline Total & 39 & $100 \%$ & 200 & $100 \%$ & 14,90 & $100 \%$ & 300 \\
\hline
\end{tabular}

La Tabla 7 muestra, según el Índice de Valor Importancia (IVI), que el Arenillo blanco es la especie de mayor importancia ecológica en el área de estudio, lo cual se sustenta al observar los valores de abundancia, frecuencia y dominancia relativos. Le sigue en orden de importancia el Pendare y finalmente el Mure. 
Tabla 8. Datos promedios de algunas variables de medición

\begin{tabular}{|l|c|c|c|c|c|c|}
\hline $\begin{array}{l}\text { Nombre } \\
\text { común }\end{array}$ & $\begin{array}{r}\text { DAP } \\
(\mathrm{m})\end{array}$ & $\begin{array}{c}\text { Calidad } \\
\text { de Fuste } \\
(1-7)\end{array}$ & $\begin{array}{c}\text { Forma } \\
\text { de copa } \\
(1-7)\end{array}$ & $\begin{array}{c}\text { Presencia } \\
\text { de lianas } \\
(1-7)\end{array}$ & $\begin{array}{c}\text { Altura } \\
\text { total } \\
(\mathrm{m})\end{array}$ & $\begin{array}{c}\text { Altura } \\
\text { fustal } \\
(\mathrm{m})\end{array}$ \\
\hline Mure & 3,32 & 1,5 & 3,2 & 1,5 & 48,97 & 25.09 \\
\hline Pendare & 4,17 & 2,0 & 3,3 & 1,1 & 47,81 & 26,60 \\
\hline Arenillo blanco & 11,5 & 1,6 & 2,3 & 1,7 & 41,96 & 23,68 \\
\hline
\end{tabular}

La Tabla 8 muestra que las especies estudiadas presentan buena calidad fitosanitaria y un alto potencial para produccion de madera; los valores del Mure y el Arenillo blanco indican que los árboles estudiados son comerciales en la actualidad, para el caso del Pendare el valor refleja que es comercial a futuro.

En cuanto al aspecto y calidad de la copa los promedios reflejan para el Arenillo blanco copas vigorosas, con círculo completo, para el Mure y el Pendare las copas de aspecto medio círculo, en todos los casos las copas están indicando un buen índice de calidad y potencial futuro de las especies. El valor entre 1 y 2 para las lianas indica baja presencia de estas, es decir ausentes y/o solo presentes en el fuste sin conexión con otros árboles, lo cual corrobora el buen estado de las tres especies estudiadas así como su sobrevivencia y producción futura de madera.

Con respecto a la altura fustal se observa que todas las especias presentan más de $20 \mathrm{~m}$ de madera aprovechable y que, si bien el Arenillo blanco es la especie de menor altura fustal, esto lo compensa con su mayor abundancia.

\section{Tasa de producción de hojarasca de las especies}

Se obtuvo la producción de hojarasca en las especies Qualea paraensis, Cedrelinga cateniformis, Coumasp., en un periodo de 15 días, durante 4 meses del año. 
Tabla 9. Aporte de hojarasca de las especies estudiadas entre 2011-2012

\begin{tabular}{|l|c|c|c|}
\hline \multicolumn{1}{|c|}{ Fecha } & $\begin{array}{c}\text { Couma sp. } \\
(\mathrm{g})\end{array}$ & $\begin{array}{c}\text { Cedrelinga } \\
\text { cateniformis } \\
(\mathrm{g})\end{array}$ & $\begin{array}{c}\text { Qualea } \\
\text { paraensis } \\
(\mathrm{g})\end{array}$ \\
\hline Mayo 2012 & 4 & 17 & 48 \\
\hline Junio 2012 & 70 & 65 & 140 \\
\hline Septiembre 2012 & 272 & 46 & 76 \\
\hline Octubre 2012 & 491 & 61 & 177 \\
\hline TOTAL & 837 & 189 & 441 \\
\hline
\end{tabular}

No se tiene registro de la estacionalidad de las especies, pero las colectas se realizaron siguiendo las sugerencias de los lugareños respecto a temporada de menor lluvia y posibilidades de encontrar frutos en los meses de mayo y junio, lo cual no se evidenció en las colectas. Según su conocimiento, la época de floración fue para los meses de enero-febrero durante los cuales no fue posible realizar colectas.

El mayor aporte en la producción de hojarasca del bosque corresponde a la especie Couma sp., el mayor aporte ocurrió en el mes de octubre de 2012, $491 \mathrm{~g}$ correspondientes al $67,35 \%$ de la hojarasca total colectada en este mes. La época de mayor caída de hojas ocurrió en el mes de octubre con 728 g $(49,69 \%)$ y septiembre $394 \mathrm{~g}(26,85 \%)$, los meses corresponden a época de invierno en la zona.

Tabla 10. Aporte de hojarasca extrapolada de las especies estudiadas

\begin{tabular}{|c|c|c|}
\hline & $\begin{array}{l}\text { Aporte de } \\
\text { hojarasca } \\
\mathbf{k g} / \mathbf{h a} / \text { ańo }\end{array}$ & $\%$ \\
\hline Mure & 479.06 & 12.88 \\
\hline Pendare & 2121.56 & 57.05 \\
\hline Arenillo blanco & 1117.81 & 30.06 \\
\hline Total & $3718.43 \mathbf{k g}$ & \\
\hline
\end{tabular}

Teniendo en cuenta que la producción de hojarasca representa un componente fundamental de la productividad primaria neta en ecosistemas arbóreos en un 
estado dinámico estable (Prause et al., 2003), el aporte de hojarasca representado por las especies $\mathbf{Q}$. paraensis, $\boldsymbol{C}$. cateniformis,Couma sp., es bastante significativo al acumular aproximadamente $3718,43 \mathrm{~kg}$ de hojarasca al año siendo Couma sp. la especie de mayor producción con 2121,56 asumiendo un $57,05 \%$ de la producción total anual, seguido de $\mathbf{Q}$. paraensis con 1117,81 y $\mathbf{C}$. cateniformis con 479,06 .

Existe una gran variabilidad en la cantidad de biomasa y el contenido de carbono acumulado en los bosques, debido a las dinámicas de hojarasca y necromasa que influencian los niveles de acumulación de esto. Verificando la anterior afirmación, en el estudio realizado de producción de hojarasca se observó que existe una diferencia significativa en la producción de hojarasca entre las épocas de muestreo, ya que la estacionalidad de la hojarasca varía y afecta las cantidades de hojarasca producida.

Debido a que, por limitaciones de tiempo y recursos, solo se realizaron 4 muestreos en el año, cada uno de 15 días, no se consideraron las fluctuaciones de aumento o disminución durante todo un año, por lo cual no es prudente extrapolar estos datos a un año, ya que generalmente estas mediciones deberían hacerse mensualmente durante un año para no incurrir en sesgos. De todas formas, deben considerarse otros factores a la baja producción de hojarasca en este caso, como son las características climáticas, edáficas, lumínicas, entre otras, que ejercen gran influencia en la producción (Agudelo, 2009). 
Tabla 11. Análisis bromatológico de las especies

\begin{tabular}{|l|c|c|c|}
\hline \multicolumn{1}{|c|}{ Análisis \% } & $\begin{array}{c}\text { Arenillo } \\
\text { blanco }\end{array}$ & Mure & Pendare \\
\hline Humedad inicial & & & \\
\hline Humedad final & 1,15 & 0,40 & 0,12 \\
\hline Cenizas & 2,4 & 1,75 & 2,82 \\
\hline Extracto etéreo (grasa) & 2,38 & 2,91 & 3,52 \\
\hline Proteína & 10,02 & 12,67 & 7,10 \\
\hline Fibra cruda & 17,97 & 21,48 & 12,57 \\
\hline Extracto no nitrogenado (calculado) & 66,07 & 60,43 & 7,87 \\
\hline Nutrientes digestibles totales (calculado) & 80,79 & 79,00 & 88,23 \\
\hline Energía bruta (calculado) & 3,97 & 4,05 & 4,05 \\
\hline Energía digestible (calculado) & 3,56 & 3,48 & 3,89 \\
\hline Energía metabolizable (calculado) & 2,92 & 2,85 & 3,19 \\
\hline
\end{tabular}

La Tabla 12 muestra el análisis que se le hizo al componente foliar de las especies estudiadas, donde se observa que las 3 representan un alto porcentaje en nutrientes digestibles, extracto no nitrogenado, fibra cruda y proteína que si bien esta parte de la planta no es de consumo humano sí es de gran importancia para la herviboría (insectos, aves, entre otros) que habita en el lugar. La grasa, la energía se encuentran en menor porcentaje sin que esto represente menos importancia para los organismos que la consumen. La ceniza es el componente con el cual se calcula la biomasa foliar, parte de uno de los objetivos presentados en el proyecto. 


\section{Biomasa foliar}

Tabla 12. Biomasa foliar de las especies estudiadas entre 2011-2012

\begin{tabular}{|c|c|c|c|c|c|}
\hline & $\begin{array}{c}\text { Peso } \\
\text { fresco } \\
(\mathrm{g})\end{array}$ & $\begin{array}{c}\text { Ceniza } \\
(\mathrm{g})\end{array}$ & $\begin{array}{c}\text { Materia } \\
\text { orgánica } \\
(\mathrm{g})\end{array}$ & $\begin{array}{c}\text { Biomasa } \\
\text { foliar } \\
(\mathbf{k g} / \mathbf{h a} / \text { año })\end{array}$ & $\%$ \\
\hline Mure & 182 & 4,52 & 177,48 & 449,86 & 12,93 \\
\hline Pendare & 833 & 15,66 & 817,34 & 2071,72 & 59,57 \\
\hline Arenillo blanco & 393 & 15,94 & 377,06 & 955,74 & 27,48 \\
\hline Total & 1408 & 36,12 & 1371,88 & 3477,32 & \\
\hline
\end{tabular}

La determinación de la biomasa foliar es importante porque enseña la distribución de la materia orgánica en el sistema y se usa con diferentes propósitos como: estimación de contenido de carbono en el bosque, cuantificación en la cantidad de nutrientes en los ecosistemas, determinación de la fijación de energía en los ecosistemas forestales, descripción cuantitativa de ecosistemas y fuentes de biomasa disponible, evaluar cambios en la estructura del bosque, cuantificación de la cantidad de gases de efecto invernadero que no se libera evitando la deforestación, y cuantificación del incremento y rendimiento de bosques y crecimiento y productividad; todos imprescindibles para comprender el ecosistema forestal, al igual que evalúan los efectos de la intervención sobre el ecosistema que repercuten en equilibrio del mismo (Mostacedo et al., 2006).

Para el caso de este estudio se observa que el Pendare es la especie con mayor aporte de biomasa foliar (59,57\%), seguida del Arenillo blanco con 27,48\% y finalmente el Mure con 14,39\%.

Biomasa aérea en el área de estudio: La biomasa aérea fue estimada a partir de los parámetros medidos en campo como altura y DAP y densidades de la madera de cada especie con la ecuación de Brown (1997) presentada en la metodología de la fase de análisis de datos.

Los datos obtenidos se presentan en la Tabla 13 [estimación de biomasa con ecuación de Brown (1997) para un área de bosque primario]. 
Tabla 13. Biomasa aérea estimada para el área de estudio

\begin{tabular}{|l|c|c|}
\hline \multicolumn{1}{|r|}{ Parámetros } & Unidades & Valores \\
\hline Volumen (V) & $\left(\mathrm{m}^{3} / \mathrm{ha}\right)$ & 88,04133 \\
\hline $\begin{array}{l}\text { Densidad fustal } \\
\text { promedio (DP) }\end{array}$ & $\left(\mathrm{t} / \mathrm{m}^{3}\right)$ & 0,62149 \\
\hline $\begin{array}{l}\text { Biomasa de } \\
\text { (BV) }\end{array}$ & $\mathrm{t} / \mathrm{ha}$ & $-190=1,74$ \\
\hline $\begin{array}{l}\text { Factor de } \\
\text { expansión } \begin{array}{l}\text { biomasa (FEB) } \\
\text { bioma del }\end{array}\end{array}$ & (t/ha) & 95,20688 \\
\hline $\begin{array}{l}\text { Biomasa total dis } \\
\text { bosque }\end{array}$ & & \\
\hline
\end{tabular}

La biomasa total para Q. paraensis, C. cateniformis, Couma sp., es de 95,20688 t/h muy similar a los valores de biomasa aérea total encontrados en bosques naturales de cuatro ecorregiones bolivianas: Amazonía, Preandino amazónico, Transición chiquitano amazónica y Chiquitanía, donde se estimaron valores de biomasa aérea total de 600 mil árboles a partir de $10 \mathrm{~cm}$ de DAP y la ecuación más exigente desarrollada por Brown et al. (1989), los cuales varían entre promedios de 97 t/ha en la ecorregión de Transición chiquitano amazónica hasta 171 t/ha en la Amazonía (Dauber et al., 2006).

Las especies C. cateniformis, Couma sp. y Q. paraensis alcanzan un gran porte (DAP promedio de $8,30 \mathrm{~m}$ y altura total promedio de $48,97 \mathrm{~m}$ para el Mure, DAP promedio de 6,95 $\mathrm{m}$ y altura promedio de $47,68 \mathrm{~m}$ para el Pendare y DAP promedio de 3,96 $\mathrm{m}$ y altura total promedio de 41,96 $\mathrm{m}$ para el Arenillo blanco), lo cual hace que tanto el volumen como la biomasa de estas especies sean considerablemente altos. Las especies con un DAP muy alto, mayores de $70 \mathrm{~cm}$, según Brown (1997), pueden equivaler a aproximadamente un $40 \%$ de la biomasa aérea en pie total, aun cuando esos árboles correspondan a menos de $5 \%$ del total de los árboles.

Quinto (2010) cuantificó la biomasa aérea en un bosque primario de Salero (Chocó biogeográfico), mediante ecuaciones diseñadas para bosques húmedos tropicales, a partir de datos de densidad de madera, diámetro (DAP) y altura de árboles medidos en dos subparcelas permanentes de 1 hectárea, las cuales se 
monitorearon en los años 1998, 2005 y 2008. La BA fue $237,31 \mathrm{t} \mathrm{ha}^{-1}, 259,99 \mathrm{t}$ ha${ }^{1}$ y $217,97 \mathrm{t} \mathrm{ha}^{-1}$ respectivamente en las subparcelas.

Chacón et al. (2007) tomaron datos de diámetro a la altura del pecho (DAP), altura y densidad en los fustales del bosque secundario de la región tropical húmeda de Costa Rica para la determinación indirecta de su volumen y el C fijado. La biomasa aérea total fue de $(99,9 \pm 15,7) \mathrm{Mg}$ ha-1. Se fijaron 46,4 Mg ha-1 de C, con una tasa de fijación de 3,1 Mg ha-1 de $\mathrm{C}$ por año.

Los valores de biomasa aérea total varían entre promedios de 97 t/ha en la ecorregión de Transición Chiquitano Amazónica hasta 171 t/ha en la Amazonía. En consecuencia, la variación del carbono almacenado es de 49 t/ha a 86 t/ha, respectivamente.

\section{Contenido de carbono en el área de estudio}

El contenido de carbono para el bosque se estimó a partir de la biomasa aérea arbórea calculada previamente, y se obtuvieron los siguientes resultados:

$$
\text { CT }=95,20688 \mathrm{t} / \mathrm{ha} * 0,5=47,60344 \mathrm{t} / \mathrm{ha}
$$

Según análisis del IDEAM (2010), sobre el contenido de biomasa y carbono potencialmente almacenado en los bosques del sistema de Parques Nacionales Naturales de Colombia, específicamente para bosques húmedos tropicales entre los que se encuentran Amacayacu, el Tuparro, La Paya, Los Katíos, Nukak, Puinawai, Tinigua, Serranía de Chiribiquete, se estimó una biomasa de 260,88 t/ha, y el valor promedio de carbono para este tipo de bosque natural 130,44 t/ha, por tal razón los estimados obtenidos en este trabajo pueden considerarse altamente representativos, si se tiene en cuenta que el área de estudio pertenece a esta clase de bh-T y que solo se trabajó sobre las especies maderables $\mathbf{Q}$. paraensis, C. cateniformis y Couma sp., las cuales representan un $36,4945 \%$ del promedio de biomasa y carbono estimados para este tipo de bosques.

Los resultados de contenido de carbono obtenidos en el estudio, fluctúan en un promedio entre los resultados obtenidos en bosques tropicales de Bolivia y Guatemala y los obtenidos en los bosques andinos de Argentina y Chile, sin embargo se deben considerar variaciones de los resultados por las diferentes metodologías empleadas en cada uno de los estudios; la cantidad de carbono dentro de estos bosques es resultado de la acción de las especies de Mure, 
Pendare y Arenillo blanco como sumideros $\mathrm{CO}_{2}$ a partir de la mitigación del $\mathrm{CO}_{2}$ de la atmósfera, prestando un servicio ambiental importante y muy necesario en esta época donde el cambio climático afecta el equilibrio ecosistémico, y por tanto la población mundial.

Según Concha et al. (2002), en 6 de 7 grupos de análisis sobre secuestro de carbono en sistemas agroforestales amazónicos, el bosque primario se ubica en el primer grupo de significancia estadística con 465,8 t por ha de carbono total superando al bosque secundario, huerto casero, silvopasturas, pastura y café con sombrío.

De acuerdo con la estimación de las reservas potenciales de carbono almacenado en la biomasa aérea en los bosques naturales de Colombia, del IDEAM (2010), se observó que en promedio la región Andina es la que mayor contenido de carbono presenta, seguida por las regiones de Orinoquia y Amazonia respectivamente, aunque en general, los contenidos de carbono entre regiones no son muy variables $\left(89,95\right.$ a $\left.95,63 \mathrm{t} \mathrm{ha}^{-1}\right)$.

Es importante tener en cuenta que, debido al significativo contenido de carbono que poseen estos bosques primarios, también tienen un alto potencial de convertirse en fuentes de emisión de $\mathrm{CO}_{2}$; en las zonas aledañas al corregimiento de Barrancominas, se ha reflejado una tendencia al cambio del uso de la tierra transformando los ecosistemas de bosque y las zonas de agricultura para dar paso principalmente a la ganadería, que en la mayoría de los casos implica la quema y la tala, esta deforestación da origen a problemas de degradación del recurso forestal, de los suelos y como consecuencia de la pérdida de biodiversidad y liberación de $\mathrm{CO}_{2}$, esto hace importante revisar el ordenamiento territorial en la región y establecer buenas prácticas de conservación y manejo del uso del suelo.

Ante los cambios atmosféricos, como el aumento en las concentraciones de $\mathrm{CO}_{2}$, el aumento de la temperatura, y la estabilidad de los parámetros meteorológicos, como la precipitación, los bosques juegan un papel regulador, esto hace sumamente importante salvaguardar los bosques y continuar no solo conservando este ecosistema Amazónico, sino además asegurando los servicios ambientales que de ellos se derivan y encaminarse hacia los objetivos planteados por el Mecanismo de Desarrollo Limpio (MDL) del Protocolo de Kyoto.

Recientemente, el IDEAM (2015) dio a conocer los cambios esperados en temperatura y precipitación a nivel nacional, regional y departamental para el 
presente siglo, lo cual permitirá al Gobierno nacional, a los entes territoriales y a los sectores productivos, planificar sus acciones de desarrollo acorde a los desafíos que presenta el cambio climático y que, según este estudio, no serán los mismos para todo el territorio; lo que significa implementar medidas de adaptación según la realidad de cada región del país.

Para esta investigación se emplearon las más recientes metodologías de vanguardia internacionales, propuestas por el Panel Intergubernamental de Cambio Climático (IPCC por sus siglas en inglés), en la que se utilizaron los modelos que mejor responden a la realidad climática de nuestro país; brindando información con mayor certidumbre para las variables de temperatura y precipitación que viviremos los colombianos en lo que queda del Siglo XXI.

\section{$[\ldots]$}

La entrega de los resultados de estos Escenarios, hacen parte de la Tercera Comunicación de Cambio Climático, herramienta que busca, adicional a registrar ante la Convención Marco de las Naciones Unidas Sobre Cambio Climático, ser la principal fuente de información y conocimiento técnico, para apoyar la toma de decisiones sobre los potenciales efectos del cambio climático en nuestro país, de modo que contribuya a la construcción de un futuro sostenible que mejore el bienestar de los colombianos. (Agroaguas S.A.S., 2015, párr. 4-6)

\section{Interrelación de especies con el ecosistema}

Las especies cumplen una función de interrelación con su medio, creando características claves dentro de un ecosistema. Un caso específico que define con claridad la función y por ende la importancia de cada especie dentro de un nicho determinado, es decir dentro de un hábitat específico y la relación con otras especies en el mismo, lo constituyen las cadenas alimenticias, jugando un papel fundamental para establecer sistemas productivos en algunos casos, y/o para regular poblaciones de especies. Es clave resaltar la importancia de la fauna, no solo desde el punto de vista estético, sino también científico, cultural y económico; en un ecosistema boscoso, de la zona del proyecto, las especies animales que viven en diferentes hábitats cumplen con funciones dentro del mismo; tal es el caso del sotobosque, en donde se desarrolla un microclima específico, creando condiciones diferentes a las que se presentan en la parte alta del bosque (dosel). 


\section{POTENCIAL CONFLICTO DE INTERESES}

No existe conflicto de intereses.

\section{FUENTES DE FINANCIACIÓN}

No se tuvo una fuente de financiación diferente a la de los autores..

\section{REFERENCIAS}

- AGROAGUAS S.A.S. (07-04-15). IDEAM: Así impactará el climático en las diferentes zonas de Colombia. Recuperado de http://www.agroaguas.com/index.php/noticias/69-ideam-asi-impactara-elcambio-climatico-en-las-diferentes-zonas-de-colombia

- Agudelo, M. (2009). Biomasa aérea y contenido de carbono en bosques de Quercus humboldtii y Colombobalanus excelsa: corredor de conservación de robles Guantiva-La Rusia-lguaque (SantanderBoyacá).Tesis Profesional. Universidad Autónoma de Occidente, Santiago de Cali, Colombia.

- Bonham, C. (1989). Measurements for terrestrial vegetation. New York: Wiley-Interscience Publication.

- Brown, S. (1997). Estimating biomass and biomass change of tropical forests: Primer. FAO Forestry Paper, 134. Roma: Food and Agriculture Organization.

- Brown, S. (2002). Measuring carbon in forest: Current status and future challenges.Enviromental Pollution, 116 (3): 363-372. 
- Brown, S., Guillespie, A. y Lugo, A. (1989). Biomass estimation methods for tropical forest with applications to forest inventory data. Forest Science, 35 (4), 881-902.

- CATIE. (1994). Curso Intensivo inventarios forestales para el bosque petenero. Flores, Petén, Guatemala. Turrialba, Costa Rica.

- Chacón, P., Leblanc, H.A. y Russo, R.O. (2007). Fijación de carbono en un bosque secundario de la región tropical húmeda de Costa Rica. Tierra Tropical, 3 (1), 1-11.

- Concha, D., Krishnamurthy, L. y Alegre, J. (2002). Secuestro de carbono en sistemas agroforestales amazónicos. Revista Chapingo, Serie Forestales y del Ambiente, 8 (2), 101-106.

- Concha, Y.J., Alegre, C.J. y Pocomucha, V. (2007). Determinación de las reservas de carbono en la biomasa aérea de sistemas agroforestales de Theobroma cacao L. en el Departamento de San Martín Perú. Tesis Profesional. Fac. Ecología Aplicada, Universidad Nacional Agraria La Molina, Lima, Perú.

- Dauber, E., Terán, J. y Guzmán, R. (2000). Estimaciones de biomasa y carbono en bosques naturales de Bolivia. Revista Forestal Iberoamericana, 1(1). Recuperado de http://www.forest.ula.ve/rforibam/archivos/DOC2.pdf

- Domínguez, E.A. (2010). Instrumentos para la medición de variables dasométricas. Fundamentos y elaboración con el alumno del ciclo formativo "Técnico Superior en Gestión y Organización de Recursos Naturales Paisajísticos". Innovación y Experiencias Educativas. Innovación y Experiencias Educativas No. 28 marzo. Aljanadic Posadas. Córdoba, Argentina, $9 \mathrm{p}$.

- Hutchinson, I. (1992). Puntos de partida y muestreo diagnóstico para la silvicultura de bosques naturales del trópico húmedo. Serie técnica. Informe Técnico No. 204. Turrialba, Costa Rica: CATIE.

- IDEAM. (2010). Resumen Ejecutivo de la Memoria Técnica de la Estimación de las reservas potenciales de carbono almacenado en la biomasa aérea en los bosques naturales de Colombia. Bogotá D.C. 
- IDEAM. (2015). Nuevos Escenarios de Cambio Climático para Colombia 2011-2100, Herramientas Científicas para la Toma de Decisiones. Bogotá D.C.: IDEAM, PNUD, MAyDS, GEF.

- Manzanero, M. (2003). Guía metodológica para el levantamiento de parcelas permanentes en la concesión forestal de AFISAP. Santa Elena, Flores, Petén, Guatemala.

- Mostacedo, B., Villegas, Z., Peña, M., Poorter, L., Licona, J. y Alarcón, A. (2006). Fijación de carbono (biomasa aérea) en áreas de manejo forestal sujetas a diferentes intensidades de aprovechamiento: implicaciones a corto y mediano plazo. Santa Cruz, Bolivia: Instituto Boliviano de Investigación Forestal (IBIF). Recuperado de http://www.mgap.gub.uy/UPACC/archivos/Informe\%20Biomasa_FAGRO.pdf

- Prause, J., Arce de Caraman, G. \& Angeloni, P. (2003). Variación mensual en el aporte de cuatro especies forestales nativas del Parque Chaqueño Humado. Revista de Ciencias Forestales, 10.

- Pedraza, H., Meléndez, C. y Muñoz, D. (s.f.). Plan de Ordenamiento Ambiental Territorial del Río Guaviare (Inírida - Barrancominas). Puerto Inírida (Guainía). Corporación para el Desarrollo Sostenible del Norte y el Oriente Amazónico.

- Pinelo, G. (2000). Manual para el establecimiento de Parcelas Permanentes de Muestreo en la Reserva de la Biosfera Maya, Petén, Guatemala. Manual Técnico No. 40. Turrialba (Costa Rica). CATIE.

- Quinto-Mosquera, H. (2010). Dinámica de la biomasa aérea de bosques primarios de Colombia y su relación con la precipitación y la altitud. Tesis Profesional. Universidad Nacional de Colombia, Sede Medellín.

- Rodríguez, G., Gil, J. y García, E. (1999). Metodología de la investigación cualitativa. Archidona, Málaga, España: Editorial ALJIBE.

- $\quad$ Rügnitz, M.T., Chacón, M.L. y Porro, R. (2009). Guía para la determinación de carbono en pequeñas propiedades rurales. 1a. Ed. Lima: Centro Mundial Agroforestal (ICRAF) / Consorcio Iniciativa Amazónica (IA). 
- Salas, J. e Infante, A. (2006). Producción primaria neta aérea en algunos ecosistemas y estimaciones de biomasa en plantaciones forestales. Revista Forestal Latinoamericana, 40, 47-70.

- Synnott, T.J. (1990). Manual de procedimientos de parcelas permanentes para el bosque húmedo tropical. Traducción: Juvenal Valerio y Gabriel Vargas. Cartago, Costa Rica: Instituto Tecnológico de Costa Rica, Departamento de Ingeniería Forestal.

- Vallejo, J., Londoño, A., López, R., Galeano, G., Álvarez, E. y Devia, W. (2005).Establecimiento de parcelas permanentes en bosques de Colombia. Serie: Métodos para estudios ecológicos a largo plazo, No. 1. Bogotá D.C.: Instituto de Investigación de Recursos Biológicos Alexander von Humboldt.

- Vargas, L. y Varela, A. (2007). Producción de hojarasca de un bosque de niebla en la reserva natural La Planada (Nariño, Colombia). Universitas Scientarium, Revista de la Facultad de Ciencias, Edición Especial I, 12, 3549.

- Villareal, H., Álvarez, M., Córdoba, S., Escobar, F., Fagua, G., Gast, F., Mendoza, H. y Umaña, A. (2004). Manual de métodos para el desarrollo de inventarios de biodiversidad. Bogotá D.C.: Instituto de Investigación de Recursos Biológicos Alexander von Humboldt.

- Zambrano, C.H., Rodríguez R.J. y Rodríguez, G.L. (2006). Estudio técnico jurídico para elevar a la categoría de Municipio el Corregimiento Departamental de Barrancominas ubicado en el Departamento del Guainía. Tesis Profesional. Fac. de Ingeniería, Universidad Distrital "Francisco José de Caldas", Bogotá D.C., Colombia.

1. Licenciada en Biología con énfasis en Educación Ambiental. Colegio Agropecuario Manuel Quintín Lame. Barrancominas, Guainía, Colombia.nubia35quiceno@gmail.com. ORCID: 0000-0001- 5186-3357 
2. Bióloga. Colegio Agropecuario Manuel Quintín Lame. Barrancominas, Guainía, Colombia.monica.tangarife@gmail.com. ORCID: 0000-0001- 7430-7660

3. Magíster en Oceanografía Biológica y Pesquera. Universidad de Manizales, Maestría en Desarrollo Sostenible y Medio Ambiente. Manizales, Colombia. ricardoalvarezleon@gmail.com. ORCID: 0000-0002- 9668-099X

Para citar este artículo: Quiceno Urbina, N.J., Tangarife Marín, G.M. y Álvarez León, R. (2015). Estimación del contenido de biomasa, fijación de carbono y servicios ambientales, en un área de bosque primario en el Resguardo Indígena Piapoco Chigüiro-Chátare de Barrancominas, departamento del Guainía (Colombia). Revista Luna Azul, 43, 171-202. Recuperado de http://200.21.104.25/lunazul/index.php?option=com_content\&view=article\&id=198

Esta obra está bajo una Licencia de Creative Commons Reconocimiento CC BY

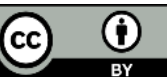

